\title{
Evaluation of a nanotechnology-based carrier for delivery of curcumin in prostate cancer cells
}

\author{
RAJESH L. THANGAPAZHAM ${ }^{1,2}$, ANU PURI $^{3}$, SHRIKANT TELE $^{3}$, \\ ROBERT BLUMENTHAL ${ }^{3}$ and RADHA K. MAHESHWARI ${ }^{1}$ \\ ${ }^{1}$ Center for Combat Casualty and Life Sustainment Research, Department of Pathology, Uniformed Services University
of the Health Sciences (USUHS), Bethesda, MD 20814, USA; ${ }^{2}$ Birla Institute of Technology and Science,
Pilani 333031, India; ${ }^{3}$ CCR Nanobiology Program, National Cancer Institute at Fredrick, NIH, MD, USA
}

Received December 3, 2007; Accepted January 25, 2008

\begin{abstract}
We have initiated studies to enhance targeted delivery of an anticancer agent, curcumin, for prostate cancer treatment by incorporating this agent into the liposomes (nanodelivery vehicles primarily composed of phospholipids) coated with prostate membrane specific antigen specific antibodies. We prepared curcumin-loaded liposomes of various lipid compositions by sonication at an average size of 100-150 nm. Un-entrapped curcumin was removed by size exclusion chromatography. Data show that curcumin preferentially partitioned into liposomes prepared from dimyristoyl phosphatidyl choline (DMPC) and cholesterol among the various compositions tested. The anti-proliferative activity of liposomal curcumin was studied using two human prostate cancer cell lines ( $\mathrm{LNCaP}$ and $\mathrm{C} 4-2 \mathrm{~B}$ ) by a tetrazolium dyebased (MTT) assay. Treatment of cells with liposomal curcumin $(5-10 \mu \mathrm{M})$ for $24-48 \mathrm{~h}$ at $37^{\circ} \mathrm{C}$ resulted in at least $70-80 \%$ inhibition of cellular proliferation without affecting their viability. On the other hand, free curcumin exhibited similar inhibition only at 10 -fold higher doses $(>50 \mu \mathrm{M})$. We also observed that $\mathrm{LNCaP}$ cells were relatively more sensitive to liposomal curcumin mediated block of cellular proliferation than $\mathrm{C} 4-2 \mathrm{~B}$ cells. We are currently developing liposome formulations with targeting ability to further improve the efficacy of curcumin in vivo.
\end{abstract}

\section{Introduction}

Turmeric (Curcuma longa Linn), is a crystalline compound which has been traditionally used in medicine and cuisine in India. Curcumin (diferuloylmethane) is the major active component of turmeric (1). Curcumin has been shown to be

Correspondence to: Dr Radha K. Maheshwari, Department of Pathology, Uniformed Services University of the Health Sciences, 4301 Jones Bridge Road, Bethesda, MD 20814, USA

E-mail: rmaheshwari@usuhs.mil

Key words: nanotechnology, curcumin, prostate cancer cancer chemopreventive in several different animal tumor bioassay systems including colon (2,3), duodenal (4), stomach (5), prostate (6) and breast (7) carcinogenesis both in vitro and in vivo. Absence of dose limiting toxicity, when curcumin is administered up to $8 \mathrm{~g}$ /day in human clinical trials, reveals the possibility of curcumin in the prevention and treatment of cancer $(8,9)$.

In spite of its promising therapeutic index, the biological activity of curcumin is severely limited due to its poor bioavailability (10). Effective methods to deliver chemotherapeutics to solid tumors and/or to increase their bioavailability have been a major challenge in current biomedical research. Nanotechnology-based tools and techniques are rapidly emerging in the fields of medical imaging and targeted drug delivery (11). Among various drug delivery vehicles, liposomes have been explored for decades due to their biodegradability and the potential to load large concentrations of therapeutic agents. Liposomes have the capacity to alter the biodistribution of drugs they encapsulate through delayed clearance and longer intravascular circulation time (12). The currently undergoing trials for various liposome formulations and the large number of commercially available therapeutics appear promising (13). Since curcumin is hydrophobic, it is considered to be a good candidate for liposome incorporation as it can be encapsulated in the lipid layer of the liposomes (14). Recent studies have shown that the incorporation of curcumin into liposomes has increased the bio-availability of curcumin significantly $(14,15)$. In a study conducted on human pancreatic carcinoma cells, the activity of liposomal curcumin was equal to or better than that of free curcumin at equimolar concentrations. Liposomal curcumin downregulated NF-кB machinery, suppressed growth, and induced apoptosis of human pancreatic cells in vitro. Antitumor and anti-angiogenic effects were also observed in vivo (15). In another study, both liposomal and HSA (human serum albumin) vehicles were examined for the transfer of curcumin to spleen lymphocyte cells of the EL4 cell line. From these studies, it was found that the liposomal vehicle was capable of loading more curcumin into cells than the human serum albumin (HSA) or the aqueous-DMSO vehicles (14).

The aim of this study was to evaluate curcumin partitioning potential into the liposomes composed of phospholipids with 


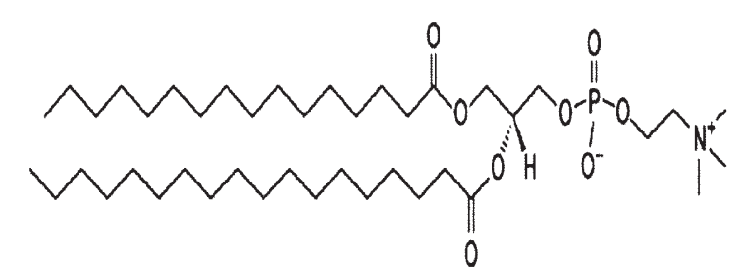

EGG PC

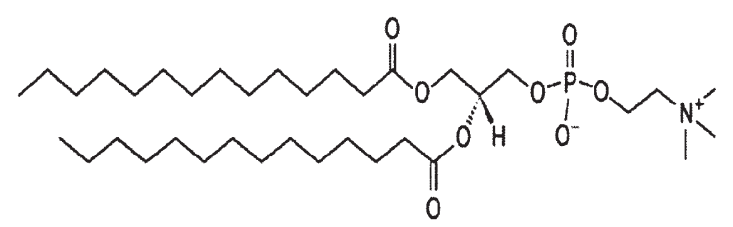

DMPC

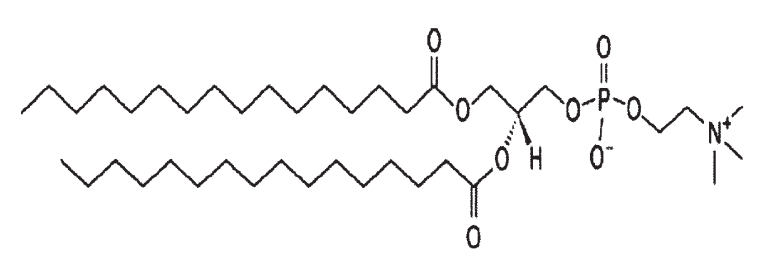

DPPC

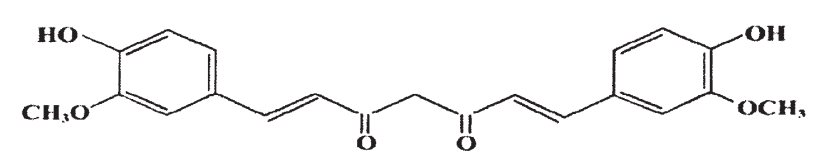

Curcumin

Figure 1. Lipid and curcumin structures. Egg phosphatidylcholine (EGG PC) cholesterol, dipalmitoyl phosphatidylcholine (DPPC) and dimyristoyl phosphatidylcholine (DMPC).

a wide range of melting transition temperatures $(\mathrm{Tm})$ and optimize conditions for encapsulating curcumin and examine the efficacy of curcumin-loaded liposome formulations on prostate cancer cells. We have chosen $\mathrm{LNCaP}$ and its isogenic more resistant derivative $\mathrm{C} 4-2 \mathrm{~B}$ as the in vitro model system. The anti-proliferative effects of free curcumin and liposomal curcumin were studied using a tetrazolium dye-based (MTT) assay. Our studies show that curcumin selectively partitions with high efficiency into DMPC liposomes as compared to DPPC or egg PC. The high therapeutic index of liposomal curcumin when compared with free curcumin shows promise for future experiments on targeted delivery.

\section{Materials and methods}

Materials. Curcumin was obtained from LKT Laboratories (St. Paul, MN), dimyristoyl phosphatidylcholine (DMPC), dipalmitoyl phosphatidylcholine (DPPC), egg phosphatidylcholine (EGG PC) cholesterol (Fig. 1) and cholesterol were obtained from Avanti Polar Lipids Inc. (Alabaster, AL). Other reagents were for Sigma-Aldrich Co. (St. Louis, MO).

Cell lines. Prostate cancer cell lines LNCaP and C4-2B cells were kind gifts from Dr Shiv Srivastava, CPDR, Rockville, MD, USA. Cultures of cells were maintained in RPMI-1640 supplemented with $10 \%(\mathrm{v} / \mathrm{v})$ heat inactivated fetal bovine serum and 1X PSN Antibiotic Mixture (VWR, Bridgeport, $\mathrm{NJ})$. Cells were cultured at $37^{\circ} \mathrm{C}$ in a humidified atmosphere of $5 \% \mathrm{CO}_{2}$ and $95 \%$ air.

Liposome preparation and curcumin encapsulation. Liposome [small unilamellar vesicles (SUV)] was prepared by probe sonication. The lipids and curcumin were mixed at a phospholipids:cholesterol:curcumin at a ratio of 90:10:10, wt:wt in chloroform in a round bottom flask and the lipid/ curcumin film was then formed by removing the solvent using a rotary evaporator. Any residual chloroform was removed by placing the films overnight in a vacuum desiccator. Multilamellar vesicles were formed by reconstituting the lipid film with HBSE buffer (10 mM HEPES, $150 \mathrm{mM} \mathrm{NaCl}, 9.1 \mathrm{mM}$ EDTA, pH 7.5) with vigorous vortexing. Unilamellar vesicles were then formed using a probe sonicator W-375 (Heat Systems-Ultrasonics, New York, USA) for 15-20 min on ice (2-min pulse with 30-sec interval between each pulse). After sonication, the liposomes were centrifuged at 2,000 x g to pellet any curcumin not intercalated into the liposomes. The liposomes were then passed through a size exclusion gel chromatography column (Bio-Rad Biogel-A5M, Bio-Rad, Hercules, CA), equilibrated with HBSE buffer, $\mathrm{pH} 7.4$, in order to separate any residual curcumin loosely associated with the liposomes. Fractions containing liposomes were pooled and filtered through $22 \mu \mathrm{m}$ filter, stored at $4^{\circ} \mathrm{C}$ and used within $48 \mathrm{~h}$ for quantitation, sizing and further analysis.

Quantification of curcumin. Liposomes containing curcumin were prepared as described above. Liposomal curcumin was quantified using a simple colorimetric assay measured at $450 \mathrm{~nm}$. A standard curve was formulated from known concentrations of curcumin HBSE-TX100 (10 mM HEPES, $140 \mathrm{mM} \mathrm{NaCl}, 4 \mathrm{mM}$ EDTA, 1\% TX-100) and was used to determine curcumin concentration in liposomes subsequent to lysis with $1 \%$ TX-100 (Fig. 2).

Measurement of liposome size and charge was conducted by dynamic light scattering techniques by the National Characterization Laboratory (Frederick, MD, USA) using a Zeta nanosizer (Malvern, UK) (data not shown).

Cell proliferation assay. $\mathrm{LNCaP}$ and $\mathrm{C} 4-2 \mathrm{~B}$ cell proliferation in the presence of various concentrations of liposomal and free curcumin was determined using the MTT (3-[4,5- 


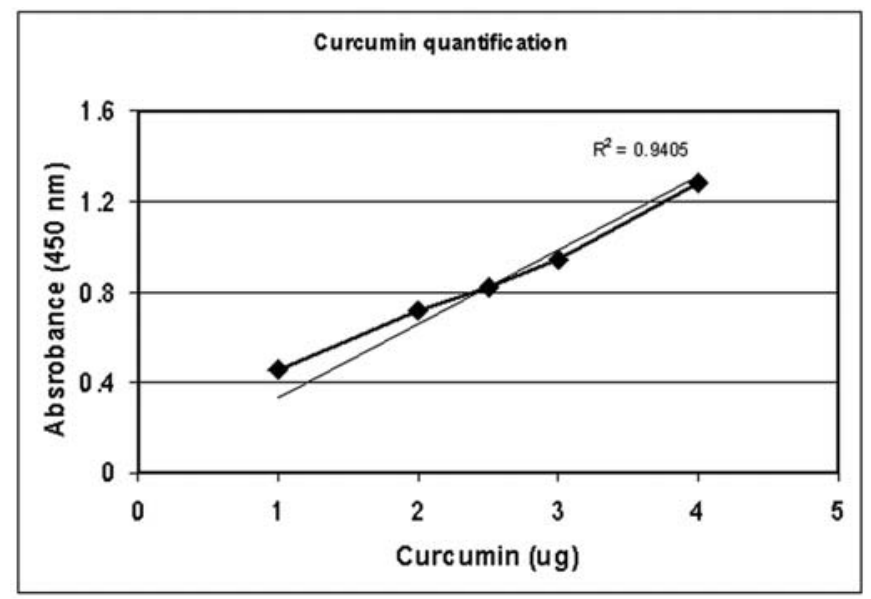

Figure 2. A standard curve for curcumin concentration was formed via a 96-well format based colorimetric assay. Known concentrations of free curcumin or liposome-entrapped curcumin were measured at $450 \mathrm{~nm}$ in HBSE-TX-100 (10 mM HEPES, 140 mM NaCl, 4 mM EDTA, 1\% TX-100) as described in Materials and methods. Using the standard curve, $20 \mu 1$ liposomes correlates to $2.5 \mu \mathrm{g}$ of curcumin to a concentration of $125 \mathrm{mg} / \mathrm{ml}$ curcumin in the liposomes.

dimethylthiazol-2-yl]-2,5-diphenyl tetrazolium bromide) cell proliferation kit (Boehringer Mannheim, Indianapolis, IN) as per manufacturer's protocol. Briefly, cells were plated in 96-well tissue culture plates in a range of 3,000 cells/well in a final volume of $100 \mu \mathrm{l}$ of medium and were allowed to attach overnight. The cells were then treated once with varying doses of liposomal and free curcumin with appropriate media control and were observed after 24 and $48 \mathrm{~h}$. After completion of the treatment, the cells were incubated with MTT for $3-4 \mathrm{~h}$ at $37^{\circ} \mathrm{C}$. Cells were lysed and the reduced intracellular formazan product was dissolved in the solubilization buffer provided in the kit. MTT is reduced to a colored water insoluble formazan salt only by metabolically active cells which is quantitated in a conventional ELISA plate reader at $570 \mathrm{~nm}$.

\section{Results}

Curcumin encapsulation efficiency. We prepared curcuminloaded liposomes of various lipid compositions by sonication to an average size of about 100-150 nm. Our initial efforts to partition curcumin into EGG PC liposomes were not successful. It was found that curcumin partitioned favorably into DMPC based liposomes. Encapsulation efficiency refers to the relative amounts of curcumin that intercalated into the liposomes. DMPC-based liposomes allowed the greatest amount of curcumin to be intercalated into the lipid membrane, whereas Egg PC-based liposomes had the lowest amount of curcumin intercalation. The curcumin intercalation efficiency into DPPC liposomes was moderate. All the formulations were made at a 1:10 curcumin:lipid ratio (w/w basis). We conclude that curcumin partition into liposome is dependent on the type of lipid used.

Curcumin quantification. Although fluorescence-based quantitation methods are available for determining curcumin concentration, we have developed a colorimetric assay based on measurement of optical density (absorbance) of curcumin at $450 \mathrm{~nm}$ in HBSE-TX100 (10 mM HEPES, $140 \mathrm{mM} \mathrm{NaCl}$, 4 mM EDTA, 1\% TX-100).

A stock solution of curcumin standard $(10 \mathrm{mg} / \mathrm{ml})$ was prepared in ethanol $(>99.9 \%)$. Immediately prior to measurement, a working dilution of standard curcumin solution $(0.5 \mathrm{mg} / \mathrm{ml})$ was prepared in HBSE-TX100. This working dilution was used to generate a standard curve using a 96-well plate format as follows: in a flat-bottom clear 96-well plate, various aliquots $(0-10 \mu 1)$ in duplicate were added to the wells. Volumes were adjusted to $0.2 \mathrm{ml}$ per well by addition of HBSE-TX100 buffer. Curcumin absorbance was measured at $450 \mathrm{~nm}$ in an ELISA plate reader (BioTek Instruments, Inc., Winooski, VT). To determine curcumin entrapment in liposomes, known volume(s) of curcumin-loaded liposomes were added to the wells, final volumes adjusted to $0.2 \mathrm{ml}$ using HBSE-TX100 and measurements conducted at $450 \mathrm{~nm}$ as desribed above. Using the standard curve, we determined $0.125 \mathrm{mg} / \mathrm{ml}$ curcumin in the liposomes. The liposomes used in this study were found to have an average particle diameter of $100 \mathrm{~nm}$ as measured by dynamic light scattering.

Cytotoxicity induced by curcumin on LNCaP and C4-2B cells. DMPC liposomal curcumin $(5-10 \mu \mathrm{M})$ treatment for $24-48 \mathrm{~h}$ at $37^{\circ} \mathrm{C}$ resulted in $70-80 \%$ inhibition of cellular proliferation. On the other hand, free curcumin exhibited similar inhibition only at 10 -fold higher doses $(>50 \mu \mathrm{M})$. It was also observed that LNCaP cells were relatively more sensitive to liposomal curcumin mediated block of cellular proliferation than $\mathrm{C} 4-2 \mathrm{~B}$ cells. Of $\mathrm{LNCaP}$ cells, 31 and $70 \%$ survived $10 \mu \mathrm{M}$ of liposomal and free curcumin treatment, whereas $\mathrm{C} 4-2 \mathrm{~B}$ cells were more resistant to liposomal treatment, surviving around 36 and $75 \%$ with $10 \mu \mathrm{M}$ of liposomal and free curcumin treatment, respectively (Fig. 3).

DPPC and DMPC liposomal curcumin had improved efficacy compared to free curcumin in inhibiting the proliferation of prostate cancer cells (Fig. 4). However, among the liposome tested, DMPC liposomal curcumin was found to be the most effective. Control liposomes showed toxic effects (10-15\%) at higher doses in our experiments which was consistent with earlier studies.

\section{Discussion}

Chemoprevention is a promising preventive measure because of its overall availability and affordability. It is conceivable that in the future patients might only need to take specifically formulated pills that prevent cancer or delay its onset. Although prostate cancer mortality has recently begun to decline in the US (2003), the cumulative costs of various treatments for early stage disease, treatment-related morbidity and treatment of biochemical failures remains substantial (16). Chemoprevention by natural products such as edible phytochemicals is a suitable alternative as an inexpensive, readily applicable, acceptable and accessible approach to cancer control and management (16). Hence, developing a phytochemical which has shown tremendous promise in vitro and preclinical animal testing in a pharmaceutically acceptable dosage form becomes pivotal. 


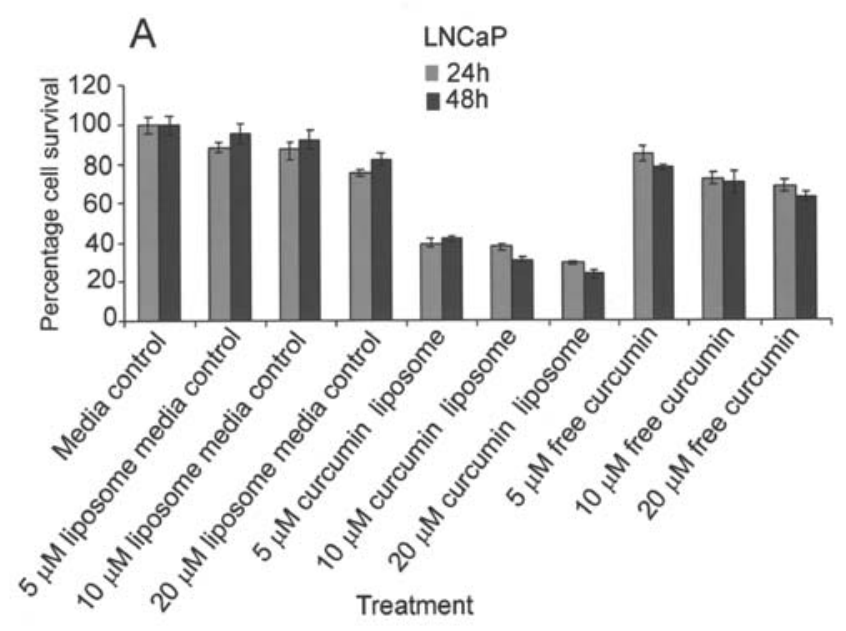

B

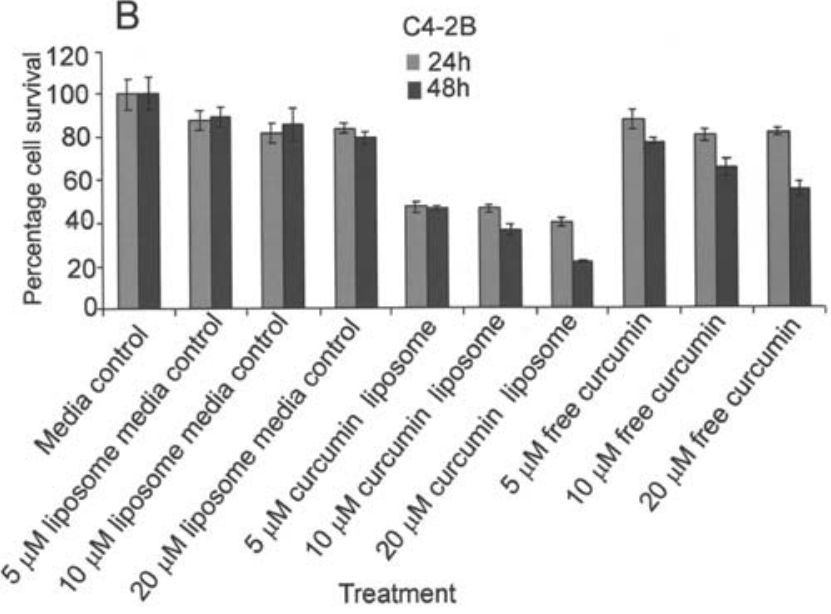

Figure 3. DMPC liposomal curcumin inhibits the proliferation of LNCaP and $\mathrm{C} 4-2 \mathrm{~B}$ incubated for 24 and $48 \mathrm{~h}$ at $37^{\circ} \mathrm{C}$. (A) Effect of different concentrations of liposomal curcumin on the proliferation of $\mathrm{LNCaP}$. (B) Effect of different concentrations of liposomal curcumin on the proliferation of $\mathrm{C} 4-2 \mathrm{~B}$. The results were compared with equivalent amount of free curcumin.

Although cancer chemoprevention studies and preclinical trials have shown curcumin as a favorable cancer chemopreventive agent in colon cancer and other gastrointestinal disorders, its efficacy in other organs is debatable due to its poor bioavailability. No curcumin was detected in the serum of human subjects administered up to $8 \mathrm{~g}$ /day of curcumin (17). Recent finding implicates rapid intestinal sulfation, glucuronidation, and reduction, especially in humans to be a plausible explanation for the poor systemic availability of curcumin (18). This situation is probably analogous to the low bioavailability of drugs, such as the oral contraceptive ethinylestradiol, which is thought to be caused by extensive sulfate conjugation. Another study from the same group clearly indicates GIT as a more approvable target for curcumin as it is significantly exposed to unmetabolized curcumin and the metabolized byproducts of curcumin have reduced ability to inhibit COX-2 expression (19). Level of curcumin and its metabolites in portal and peripheral blood, bile and liver tissue as measured by HPLC was found to be so low that it was unlikely to exert pharmacological activity in
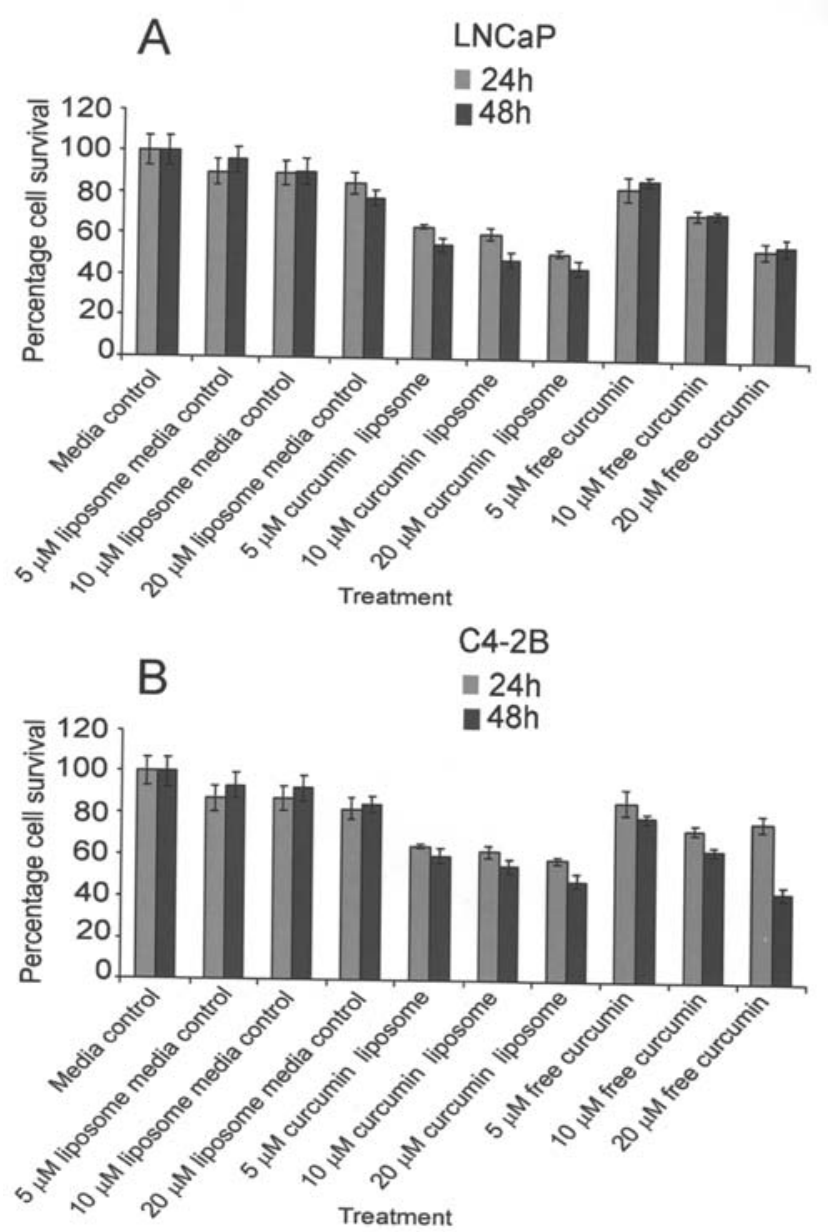

Figure 4. DPPC liposomal curcumin inhibits the proliferation of LNCaP and $\mathrm{C} 4-2 \mathrm{~B}$ incubated for 24 and $48 \mathrm{~h}$ at $37^{\circ} \mathrm{C}$. (A) Effect of different concentrations of liposomal curcumin on the proliferation of LNCaP. (B) Effect of different concentrations of liposomal curcumin on the proliferation of C4-2B. The results were compared with equivalent amount of free curcumin.

those human tissues (20). It becomes critical for the drug to reach the infection site and attain a pharmacologically desired concentration to be considered a potential drug candidate.

Hence, it becomes pivotal to develop a suitable delivery agent and ways to increase the bioavailability of curcumin to take advantage of curcumin's chemopreventive potential to various affected organs. Research is being pursued to increase the bioavailability of curcumin by various other means such as formulating curcumin with phosphatidylcholine and soyphospholipids $(21,22)$.

Previous studies have reported successful use of various chemotherapeutic agents delivered in a liposome formulation (23-25). In this study, we have considered the use of liposomes as a delivery model for curcumin. The results from the cell proliferation assays provide strong evidence for liposomes as effective nanodelivery vehicles that increase the bioavailability of curcumin. Currently, strategies to conjugate liposome formulations with prostate membrane specific antigen (PMSA) are being developed such that they can 
specifically target the prostate cancer cells to further improve efficacy of curcumin in vivo.

\section{Acknowledgements}

This work was supported by intramural grant from the Uniformed Services University of the Health Science, Bethesda and US-INDIA Foreign Currency Fund from US Department of State to USUHS. This research was supported (in part) by the intramural research program of the NIH, NCI, Center for Cancer Research. The authors are grateful to Mr. Anuj Sharma for his help and valuable suggestions. The opinions or assertions contained herein are the private views of the authors and should not be construed as official or necessarily reflecting the views of the Uniformed Services University of the Health Sciences or the Department of Defense, USA.

\section{References}

1. Ammon HP and Wahl MA: Pharmacology of Curcuma longa. Planta Med 57: 1-7, 1991.

2. Perkins S, Verschoyle RD, Hill K, Parveen I, Threadgill MD, Sharma RA, Williams ML, Steward WP and Gescher AJ: Chemopreventive efficacy and pharmacokinetics of curcumin in the $\mathrm{min} /+$ mouse, a model of familial adenomatous polyposis. Cancer Epidemiol Biomarkers Prev 11: 535-540, 2002.

3. Chen A, Xu J and Johnson AC: Curcumin inhibits human colon cancer cell growth by suppressing gene expression of epidermal growth factor receptor through reducing the activity of the transcription factor Egr-1. Oncogene 25: 278-287, 2006.

4. Huang MT, Lou YR, Ma W, Newmark HL, Reuhl KR and Conney AH: Inhibitory effects of dietary curcumin on forestomach, duodenal and colon carcinogenesis in mice. Cancer Res 54: 5841-5847, 1994.

5. Singh SV, Hu X, Srivastava SK, Singh M, Xia H, Orchard JL and Zaren HA: Mechanism of inhibition of benzo[a]pyreneinduced forestomach cancer in mice by dietary curcumin. Carcinogenesis 19: 1357-1360, 1998.

6. Choudhuri T, Pal S, Das T and Sa G: Curcumin selectively induces apoptosis in deregulated cyclin D1-expressed cells at G2 phase of cell cycle in a p53-dependent manner. J Biol Chem 280: 20059-20068, 2005.

7. Cheng AL, Hsu CH, Lin JK, Hsu MM, Ho YF, Shen TS, Ko JY, Lin JT, Lin BR, Ming-Shiang W, Yu HS, Jee SH, Chen GS, Chen TM, Chen CA, Lai MK, Pu YS, Pan MH, Wang YJ, Tsai CC and Hsieh CY: Phase I clinical trial of curcumin, a chemopreventive agent, in patients with highrisk or pre-malignant lesions. Anticancer Res 21: 2895-2900, 2001.

8. Aggarwal BB, Kumar A and Bharti AC: Anticancer potential of curcumin: preclinical and clinical studies. Anticancer Res 23: 363-398, 2003.

9. Kostarelos K, Emfietzoglou D, Papakostas A, Yang WH, Ballangrud AM and Sgouros G: Engineering lipid vesicles of enhanced intratumoral transport capabilities: correlating liposome characteristics with penetration into human prostate tumor spheroids. J Liposome Res 15: 15-27, 2005.

10. Caruthers SD, Wickline SA and Lanza GM: Nanotechnological applications in medicine. Curr Opin Biotechnol 18: 26-30, 2007.
11. Dorai T, Cao YC, Dorai B, Buttyan R and Katz AE: Therapeutic potential of curcumin in human prostate cancer. III. Curcumin inhibits proliferation, induces apoptosis and inhibits angiogenesis of LNCaP prostate cancer cells in vivo. Prostate 47: 293-303, 2001.

12. Allen TM: Liposomes. Opportunities in drug delivery. Drugs 54 (Suppl 4): 8-14, 1997

13. Goyal P, Goyal K, Vijaya Kumar SG, Singh A, Katare OP and Mishra DN: Liposomal drug delivery systems - clinical applications. Acta Pharm 55: 1-25, 2005.

14. Kunwar A, Barik A, Pandey R and Priyadarsini KI: Transport of liposomal and albumin loaded curcumin to living cells: an absorption and fluorescence spectroscopic study. Biochim Biophys Acta 1760: 1513-1520, 2006.

15. Li L, Braiteh FS and Kurzrock R: Liposome-encapsulated curcumin: in vitro and in vivo effects on proliferation, apoptosis, signaling and angiogenesis. Cancer 104: 1322-1331, 2005.

16. Klein EA and Thompson IM: Update on chemoprevention of prostate cancer. Curr Opin Urol 14: 143-149, 2004.

17. Lao CD, Ruffin MT, Normolle D, Heath DD, Murray SI, Bailey JM, Boggs ME, Crowell J, Rock CL and Brenner DE: Dose escalation of a curcuminoid formulation. BMC Complement Altern Med 6: 10, 2006.

18. Ireson CR, Jones DJ, Orr S, Coughtrie MW, Boocock DJ, Williams ML, Farmer PB, Steward WP and Gescher AJ: Metabolism of the cancer chemopreventive agent curcumin in human and rat intestine. Cancer Epidemiol Biomarkers Prev 11: 105-111, 2002.

19. Ireson C, Orr S, Jones DJ, Verschoyle R, Lim CK, Luo JL, Howells L, Plummer S, Jukes R, Williams M, Steward WP and Gescher A: Characterization of metabolites of the chemopreventive agent curcumin in human and rat hepatocytes and in the rat in vivo and evaluation of their ability to inhibit phorbol esterinduced prostaglandin E2 production. Cancer Res 61: 1058-1064, 2001

20. Garcea G, Jones DJ, Singh R, Dennison AR, Farmer PB, Sharma RA, Steward WP, Gescher AJ and Berry DP: Detection of curcumin and its metabolites in hepatic tissue and portal blood of patients following oral administration. Br J Cancer 90: 1011-1015, 2004.

21. Liu A, Lou H, Zhao L and Fan P: Validated LC/MS/MS assay for curcumin and tetrahydrocurcumin in rat plasma and application to pharmacokinetic study of phospholipid complex of curcumin. J Pharm Biomed Anal 40: 720-727, 2006.

22. Marczylo TH, Verschoyle RD, Cooke DN, Morazzoni P, Steward WP and Gescher AJ: Comparison of systemic availability of curcumin with that of curcumin formulated with phosphatidylcholine. Cancer Chemother Pharmacol 60: 171-177, 2007.

23. Vaage J, Donovan D, Loftus T, Abra R, Working P and Huang A: Chemoprevention and therapy of mouse mammary carcinomas with doxorubicin encapsulated in sterically stabilized liposomes. Cancer 73: 2366-2371, 1994.

24. Webb MS, Johnstone S, Morris TJ, Kennedy A, Gallagher R, Harasym N, Harasym T, Shew CR, Tardi P, Dragowska WH, Mayer LD and Bally MB: In vitro and in vivo characterization of a combination chemotherapy formulation consisting of vinorelbine and phosphatidylserine. Eur J Pharm Biopharm 65: 289-299, 2007.

25. Hwang TL, Lee WR, Hua SC and Fang JY: Cisplatin encapsulated in phosphatidylethanolamine liposomes enhances the in vitro cytotoxicity and in vivo intratumor drug accumulation against melanomas. J Dermatol Sci 46: 11-20, 2007. 